\title{
Overview of the Phoenix Entry, Descent and Landing System Architecture
}

\author{
Myron R. Grover III $^{1}$ and Benjamin D. Cichy ${ }^{2}$ \\ Jet Propulsion Laboratory, California Institute of Technology, Pasadena, CA, 91109, USA \\ Prasun N. Desai ${ }^{3}$ \\ NASA Langley Research Center, Hampton, VA, 23681, USA
}

\begin{abstract}
NASA's Phoenix Mars Lander began its journey to Mars from Cape Canaveral, Florida in August 2007, but its journey to the launch pad began many years earlier in 1997 as NASA's Mars Surveyor Program 2001 Lander. In the intervening years, the entry, descent and landing (EDL) system architecture went through a series of changes, resulting in the system flown to the surface of Mars on May 25th, 2008. Some changes, such as entry velocity and landing site elevation, were the result of differences in mission design. Other changes, including the removal of hypersonic guidance, the reformulation of the parachute deployment algorithm, and the addition of the backshell avoidance maneuver, were driven by constant efforts to augment system robustness. An overview of the Phoenix EDL system architecture is presented along with rationales driving these architectural changes.
\end{abstract}

\begin{tabular}{llll}
\multicolumn{3}{c}{ Nomenclature } \\
BAM & $=$ Backshell avoidance maneuver & MCO & $=$ Mars Climate Orbiter \\
CFD & $=$ Computational fluid dynamics & MER & $=$ Mars Exploration Rover \\
DGB & $=$ Disk gap band & MOLA & $=$ Mars Orbiter Laser Altimeter \\
EDL & $=$ Entry, descent, and landing & MPL & $=$ Mars Polar Lander \\
EGSE & $=$ Electronic ground support equipment & MSP & $=$ Mars Surveyor Program \\
HS & $=$ Heatshield & RCS & $=$ Reaction control system
\end{tabular}

\section{Introduction}

$\mathrm{O}$ May $25^{\text {th }}, 2008$ at 23:38 UTC, the Phoenix Mars Lander made the first successful powered landing on Mars since the Viking landings three decades earlier. ${ }^{1}$ The eruption of cheers and applause in the control room following the first indication of a successful touchdown were more than ten years in the making, having begun in 1997 during a different, faster, better, cheaper era of planetary space flight. Originally born as NASA's Mars Surveyor Program 2001 Lander, the spacecraft and mission lived through the assembly and test phase, but then was canceled following the successive losses of its cousin spacecraft the Mars Climate Orbiter (MCO) and Mars Polar Lander (MPL) in 1999. Lying essentially dormant and partially complete in storage at the location of its creation, the Lockheed Martin Space Systems facility in Littleton, Colorado, it literally rose from the ashes of the Mars Surveyor Program when it was reborn as Phoenix in 2003, as part of NASA's Mars Scout Program.

Through the years from the inception of the Mars 2001 Lander to the landing of the spacecraft as Phoenix, the design of the entry, descent and landing (EDL) architecture, as well as the spacecraft itself, evolved in response to changing mission design and focused efforts to enhance spacecraft system robustness. What follows in this paper is a chronicling of the evolution of the design and design drivers, as well as an overview of the entry, descent and landing architecture that provided the historic first landing in the northern polar region of Mars.

\footnotetext{
${ }^{1}$ Senior Aerospace Engineer, EDL Systems Advanced Technologies Group, 4800 Oak Grover Drive, MS 264-623, myron.r.grover@jpl.nasa.gov, AIAA Member.

${ }^{2}$ Senior Aerospace Engineer, Guidance \& Control Software \& Flight Software Testing, 4800 Oak Grover Drive, MS 264-623, benjamin.d.cichy@jpl.nasa.gov.

${ }^{3}$ Senior Aerospace Engineer, Atmospheric Flight \& Entry Systems Branch, MS 489, prasun.n.desai@nasa.gov, AIAA Associate Fellow.
} 


\section{Basic Elements of Phoenix EDL System}

The basic elements of the Phoenix EDL system remain unchanged from the original Mars 2001 Lander. As shown in Fig. 1, the spacecraft arrives at Mars in the Pre-Entry Configuration, which consists of a cruise stage and entry vehicle. The cruise stage, which provides solar power and navigation functions during the cruise to Mars, is jettisoned prior to atmospheric entry, leaving the Entry Configuration. More than $99 \%$ of the Mars relative energy possessed by the vehicle is removed via entry configuration atmospheric drag during hypersonic and supersonic flight. At target deploy conditions, a Viking heritage disk-gap-band (DBG) supersonic parachute is deployed. This Parachute Configuration further reduces the energy of the EDL system, while carrying out key activities in preparation for terminal descent. These activities include jettison of the entry vehicle heatshield (HS) exposing the lander inside the entry vehicle. Heatshield jettison is followed by lander leg deploy and radar power on. The radar is used for both altimetry and velocimetry and is a key element of the landing system. This configuration is shown in Fig. 1 as the Post HS \& Leg Deploy Configuration. Upon reaching a desired velocity/altitude state, the lander separates from the parachute/backshell and begins using a system of 12 pulsed thrusters to perform a gravity turn powered descent. Touchdown and engine cutoff occurs when one of three landing legs contacts the ground. The Terminal Descent Configuration is the fifth illustration shown in Fig.

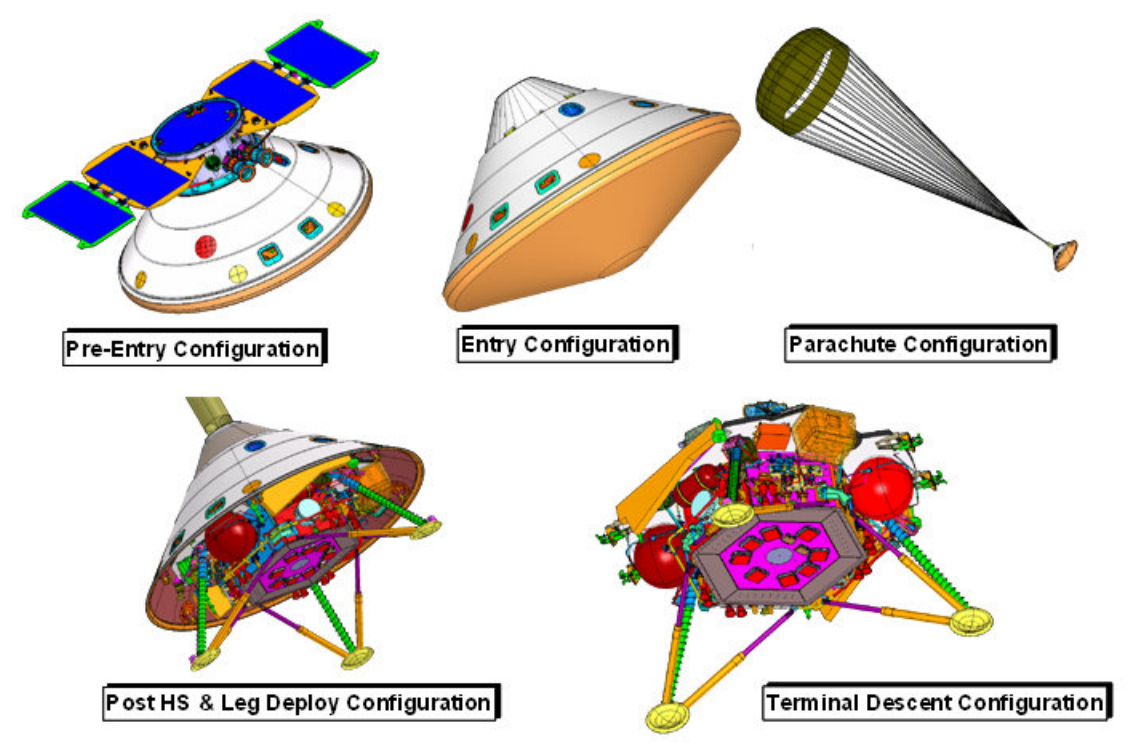

Figure 1. Basic elements of the Phoenix entry, descent and landing system.

\section{Mars 2001 Lander vs. Phoenix: Mission Design Differences}

The original Mars 2001 Lander mission design differed from the Phoenix mission design in a number of key ways. Orbital mechanics of the 2001 launch opportunity led to a Mars 2001 Lander inertial atmospheric entry velocity of $7.0 \mathrm{~km} / \mathrm{s}$. The Phoenix launch opportunity led to a less energetic and less stressing inertial entry velocity of $5.6 \mathrm{~km} / \mathrm{s}$. The science goals of the two missions also led to two very different landing regions. While the Mars 2001 Lander was planned to target the equatorial region of Mars, the Phoenix landing site is on the northern plains of Mars. This difference resulted in a significant difference in landing site elevation between the two missions. The Mars 2001 Lander was required to land at a highest elevation of $+2.5 \mathrm{~km}$ relative to the MOLA reference areoid, more than any previous mission, while the Phoenix landing site is at $-4.1 \mathrm{~km}$ relative to the MOLA areoid. A difference of more than $6 \mathrm{~km}$ provided Phoenix a more comfortable EDL timeline. Finally, because of the relative homogeneity and benign character of the Phoenix landing site and to reduce EDL complexity, the use of hypersonic guidance in the original 2001 design to reduce landed footprint size was descoped for Phoenix. Table 1 provides a list of defining differences between the two mission EDL architectures. In the following sections, the process and design drivers that led to the final Phoenix design are described in more detail. 
Table 1. Comparison of 2001 Mars Lander and Phoenix EDL Architectures.

\begin{tabular}{|l|c|c|}
\hline EDL Architectural Feature & $\begin{array}{c}\text { 2001 Mars Lander EDL } \\
\text { Design Value }\end{array}$ & $\begin{array}{c}\text { Phoenix EDL } \\
\text { Design Value }\end{array}$ \\
\hline Inertial Entry Velocity & $7.0 \mathrm{~km} / \mathrm{s}$ & $5.6 \mathrm{~km} / \mathrm{s}$ \\
Inertial Entry Flight Path Angle & $-12.5^{\circ}$ & $-13.0^{\circ}$ \\
Hypersonic/Supersonic Flight & Apollo Guidance \\
Peak Heating & $79 \mathrm{~W} / \mathrm{cm}^{2}$ & Ballistic Trajectory \\
Peak Deceleration & 10.5 Earth G's & $46.1 \mathrm{~W} / \mathrm{cm}^{2}$ \\
Parachute Deploy Dynamic Pressure & $625 \mathrm{~Pa}$ & 9.3 Earth G's \\
Parachute Deploy Altitude & $10.2 \mathrm{~km}$ & $490 \mathrm{~Pa}$ \\
Time on Parachute & $117 \mathrm{~s}$ & $12.9 \mathrm{~km}$ \\
Lander Separation Height & $1040 \mathrm{~m}$ & $173 \mathrm{~s}$ \\
Length of EDL Phase & $369 \mathrm{~s}$ & $940 \mathrm{~m}$ \\
Landing Site Location & Equatorial & $440 \mathrm{~s}$ \\
Max. Landing Site Elevation (w.r.t MOLA) & $+2.5 \mathrm{~km}$ & Polar \\
\hline
\end{tabular}

\section{The Evolution of the Phoenix EDL Architecture}

The following section describes the key evolutionary steps in the design of the Phoenix EDL architecture, beginning with the design originating from the Phoenix proposal, and concluding with the design actually flown to Mars. Having lineage to the lost Mars Polar Lander, the very first Phoenix EDL design drivers were rooted in the findings of the Mars Polar Lander Failure Review Board and the 2001 Mars Lander Return to Flight recommendations. These included addressing a touchdown indicator flight software bug that was identified as the most probable cause of the MPL failure. Additionally, design changes to the radar were also identified and incorporated into the original Phoenix EDL design, and EDL UHF communication capability was also added, as were other more minor changes all of which were targeted at enhancing the robustness of the Phoenix EDL system.

\section{A. Phoenix EDL Architecture at Inception}

At the beginning of the Phoenix project, the EDL architecture resembled strongly the Mars 2001 Lander architecture, with the major changes being inertial entry velocity and landing site elevation. Hypersonic guidancewas part of the Phoenix baseline at the inception of the project. Figure 2 shows the baseline Phoenix EDL architecture as it existed in late 2004.

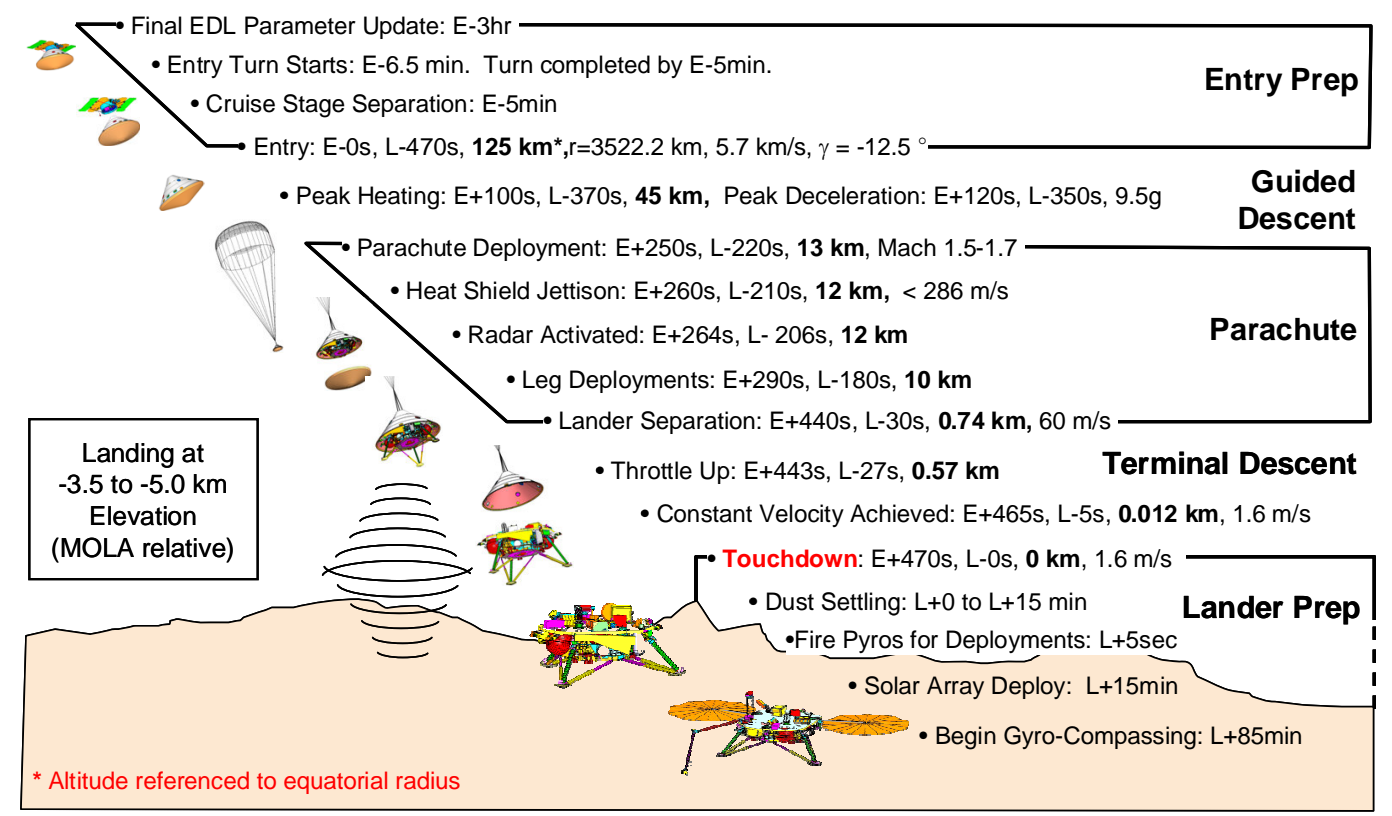

Figure 2. Phoenix EDL architecture and sequence of events as it existed in late 2004. 


\section{B. Changes to Parachute Deploy Dynamic Pressure, Parachute Diameter, and Parachute Deploy Trigger}

The original Mars 2001 Lander parachute deployment trigger was a velocity trigger that used Mars relative velocity propagated from entry minus 10 minutes using onboard accelerometers. When the trigger velocity condition was achieved, the parachute was deployed. An assessment early in the Phoenix project of the loads on the lander at parachute deployment showed low or negative margin. Because the loads at parachute deployment are proportional to dynamic pressure, an effort was undertaken to reduce the dynamic pressure condition at parachute deployment.

The first step in the dynamic pressure reduction was a simple reduction in a target parachute deployment velocity. By reducing the velocity, the dynamic pressure is also reduced; however, there is a limiting factor to this approach. Pushing back on deploy velocity reduction is the desire to avoid being at or near a transonic condition at parachute deployment. As the entry vehicle approaches the parachute deployment velocity state, it is in supersonic flight below Mach 2. The aerodynamic behavior of the blunt body vehicle enters a dynamic instability region as it approaches Mach 1. This aerodynamic instability causes the capsule angle of attack to oscillate at a growing rate. Two considerations come into play with this growing oscillation: parachute qualification tests and vehicle dynamics after parachute deploy. The Phoenix Viking heritage DBG supersonic parachute was qualified to deploy at angles of attack at or less than $13^{\circ}$ (from Viking flights), therefore the parachute must be deployed prior to oscillations of this magnitude. Equally dependant on parachute deploy angle of attack magnitude is the excitation of an oscillation mode dubbed the "wrist mode". The wrist mode is the oscillation of the lander about the parachute attachment point while hanging below the parachute. A parachute deployment at a large angle of attack causes the application of a large load on the lander in a direction not aligned with the lander center of mass. This orientation leads to a large torque on the vehicle at parachute deployment and produces an undesirable high rate wrist mode following parachute deployment. Both parachute qualification limits and wrist mode excitation bound the extent to which parachute deployment velocity can be lowered. Due to these constraints, one additional modification was made to increase the structural margin of the lander: the parachute diameter was decreased from $13.41 \mathrm{~m}$ to $11.73 \mathrm{~m}$ to further reduce the loads imparted onto the lander.

Having both lowered the target parachute deployment velocity, thereby lowering the parachute dynamic pressure, and reduced the parachute diameter, an effort was also undertaken to reduce the dynamic pressure dispersion at parachute deploy. Through Monte Carlos analysis, dispersion of parachute deployment dynamic pressure can be assessed as a function of dispersion of atmospheric density, accelerometer performance and knowledge of entry vehicle drag. All of these dispersions and uncertainties lead to a dispersion of the parachute deployment dynamic pressure. It was determined through this analysis that a parachute trigger based on acceleration, rather than velocity, was capable of producing a smaller dynamic pressure dispersion at parachute deployment. Because dynamic pressure is directly proportional to acceleration, acceleration can be used as a measurement of dynamic pressure, with uncertainties incorporated for vehicle mass and vehicle drag. This approach was used successfully on the Mars Exploration Rover landings. Thus, Phoenix incorporated an acceleration trigger into its parachute deployment trigger, so that the final resulting parachute deployment trigger flown by Phoenix was a hybrid trigger using either acceleration or velocity as a trigger point, but tuned so that statistically the trigger was very likely to deploy on acceleration. This implementation allowed the parachute deploy dynamic pressure condition to be both lower and tighter in dispersion, thereby reducing loads on the lander at deployment while keeping the deployment state comfortably away from the transonic region.

\section{Removal of Hypersonic Guidance}

A modified version of the Apollo hypersonic guidance system used by the Apollo command module during earth re-entry was part of the Mars 2001 Lander design. Incorporated as a technology demonstration, the Mars 2001 Lander development simulations showed the possibility of achieving a $10 \mathrm{~km}$ landed footprint, much smaller than a landed footprint for an unguided, ballistic entry. Under the ambitious timeline of the Mars Surveyor Program, the Mars 2001 Lander would be flown just prior to precursor missions for Mars Sample Return that would require precision landing made possible by hypersonic guidance. It thus was reasonable that the 2001 Mars Lander should demonstrate hypersonic guidance for the first time at Mars.

When the spacecraft was reborn as Phoenix, the ambitions of the Mars Surveyor Program had subsequently been discarded being replaced by a new Mars exploration timeline. The rationale for Phoenix to retain hypersonic guidance capability thus became ambiguous, and such was the state at the beginning of the Phoenix project. Driven in large part by design momentum from the Mars 2001 Lander project, the Phoenix project retained hypersonic guidance for more than a year of its existence. However, there were two main considerations that eventually led to the capability being discarded: design complexity and landing site characterization. 
As part of the Mars Scout Program, Phoenix was carried out under a limited, fixed cost budget. In such a situation, the development of a new, complex capability like hypersonic guidance was difficult to characterize leading to significant cost risk. While implementation was fairly straightforward, the approach to adequately test and verify the capability was not fully understood. Combined with concurrent landing site characterization work showing a relatively homogeneous and benign landing region, both the lack of need for and practicality of hypersonic guidance led to its removal from the Phoenix EDL architecture in 2005.

Having removed hypersonic guidance, the Phoenix engineering team was left with the question of what would serve as an optimal and robust replacement. Because the vehicle was designed for hypersonic guidance, it had incorporated in its design a center of mass offset. This offset produced a trim angle of attack of about $3^{\circ}$ that generated lift, the direction of which was steered by the guidance system to produce downtrack/crosstrack maneuvering. With the guidance system descoped, the engineering team had the option to retain the vehicle trim angle and lift (similar to Viking), or rebalance the vehicle and change it into a purely ballistic spacecraft. A trade study was undertaken to assess new baseline options, the outcome of which was to change the hypersonic/supersonic phase of the EDL architecture into a lifting trajectory. This first evolutionary step is shown in Fig. 3. This choice retained the vehicle center of mass offset and fixed the resulting lift in an upward direction, and was deemed desirable by much of the engineering team in part because it resulted in the smallest design change while removing the complexities of hypersonic guidance. It also had the benefit of raising the Mach number at parachute deployment, thereby operating the system further away from the transonic region and the aerodynamic instability. A significant down side of the design decision was a large growth in landed footprint to $250 \mathrm{~km}$. This can also be seen in Fig. 3. The lifting architecture was retained with the knowledge that concern about surface landing hazards might eventually drive the need for footprint reduction, and indeed this did happen in late 2005.

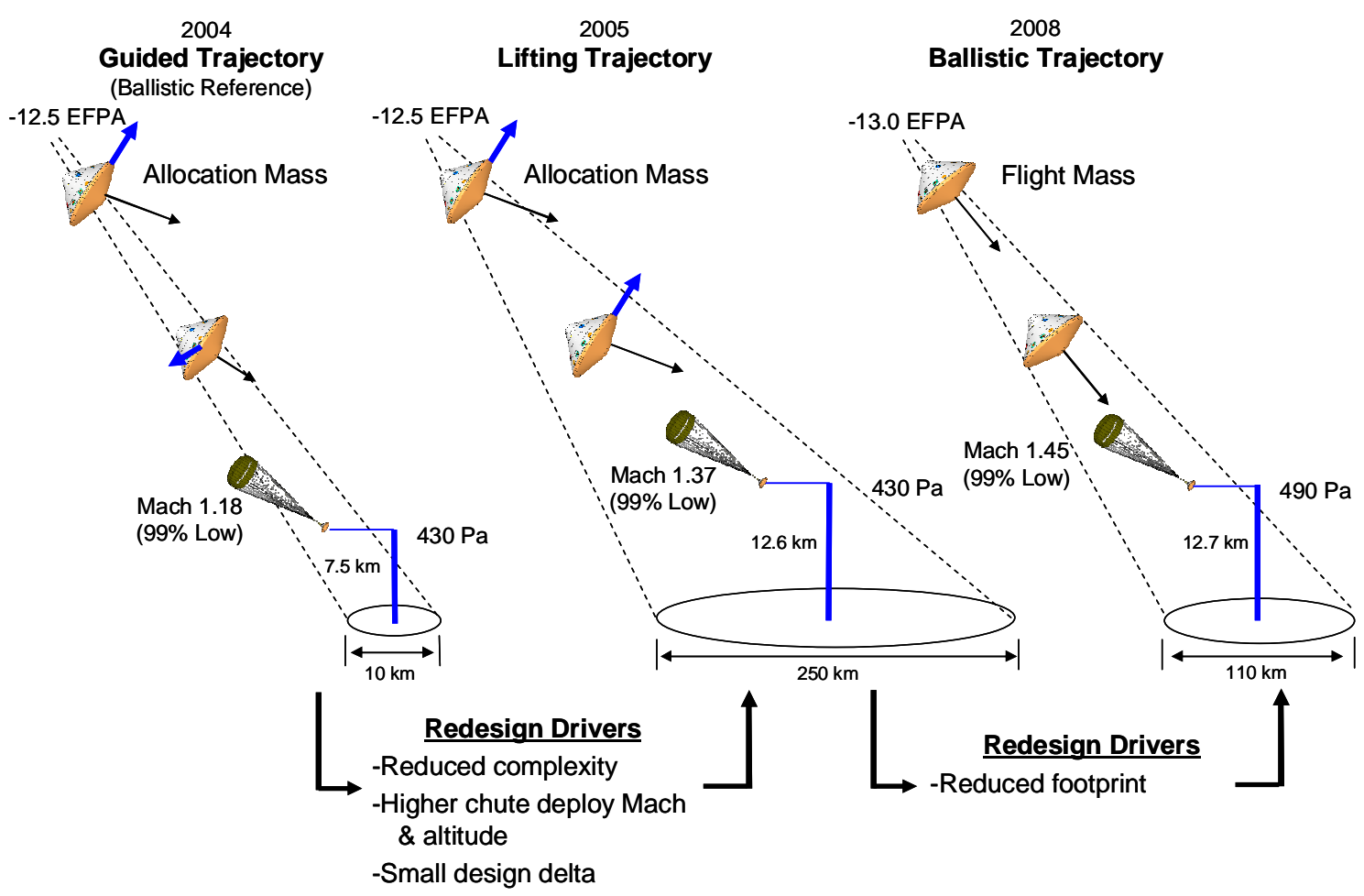

Figure 3. Evolution of the Phoenix EDL hypersonic/supersonic phase.

Given the directive to reduce landed footprint, another trade study was undertaken to do a fresh assessment of not only trajectory options, but also entry flight path angle options. Fig. 4 illustrates the formulation of the trade study. A performance trade space was established with dimensions in entry flight path angle and trajectory type. A follow-on implementation trade space assessed attitude control options, whether a spinning entry vehicle as was the case for the Mars Exploration Rovers, or a 3-axis actively stabilized vehicle as was the case for Viking and MPL. The results of the trade study established a 3-axis stabilized ballistic entry at a slightly steepened $-13.0^{\circ}$ entry flight path angle as the Phoenix baseline. This design remained the baseline for the remainder of the project and was the 
architecture flown on landing day. It provided an adequately reduced landed footprint size (approx. $110 \mathrm{~km}$ ), while providing sufficient margin and risk balance. This final evolutionary step is shown in Fig. 3.

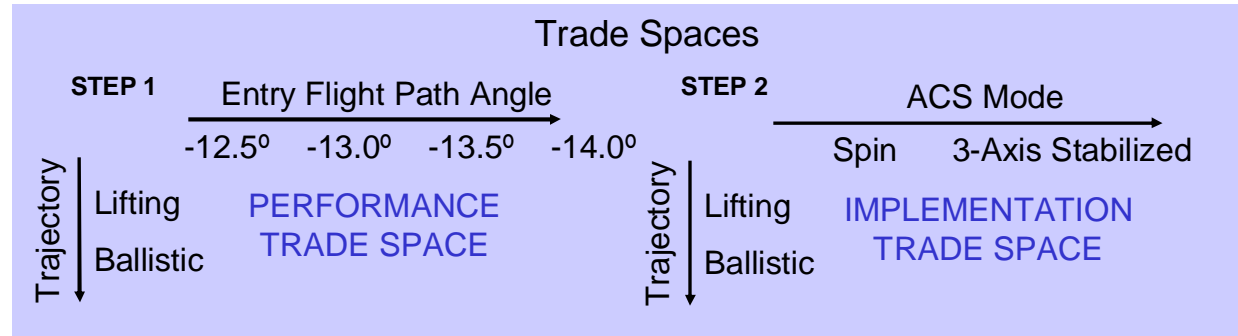

Figure 4. EDL architecture trade space that established the as-flown baseline design.

\section{Terminal Descent and Augmentation of Landing Radar}

Once the parachute has been successfully deployed, the lander must autonomously decide when to separate from the parachute and begin propulsive descent. This event is a critical decision with little margin for error. If separation occurs too early in the trajectory, the lander will run out of propellant prior to reaching the surface of Mars. If separation occurs too late in the trajectory, the lander will not have enough time to slow down to a safe touchdown velocity. To further complicate matters, the propagated entry state used to deploy the parachute was not accurate enough to make this separation decision. Instead the lander must use another sensor - a landing radar - to provide the altitude and velocity knowledge required for a successful separation and touchdown. The performance of this sensor thus becomes critical to the success of the mission.

The landing radar used by Phoenix was originally selected by the Mars Survey Program (MSP) for the Mars Polar Lander (MPL) mission, and augments a commercial F-16 altimeter with a velocimeter and an array of electronically steerable antennas. The radar provides altitude and three-axis velocity measurements from approximately $2 \mathrm{~km}$ to 15 meters above the surface.

The Phoenix mission identified early on the need for additional testing of the MSP-derived landing radar. The performance of the radar was a prime failure mode identified in the MPL design, and as such received considerable attention by the Phoenix EDL team. A wide ranging test program was performed, using three complementary test venues: helicopter field tests with a flight-spare radar, bench-level Electronic Ground Support Equipment (EGSE) tests with the flight radar, and a high-fidelity computer simulation of the integrated system. While it is impossible to test the radar on Earth in the same environment it will experience on landing day, the combinations of these test venues allowed the team to perform an unprecedented analysis of the radar performance.

The test program uncovered many problems and idiosyncrasies with the radar performance, most with straightforward mitigations. However, in December of 2006, a serious problem was discovered using the highfidelity software simulation.

Under the original EDL timeline, shown in Fig. 2, the radar begins searching for the ground $4 \mathrm{sec}$ after the separation of the heatshield. The team had long been aware that due to the close proximity of the heatshield at radar power on, the radar may initially lock onto the heatshield yielding erroneous measurements. In response, the Phoenix Flight Software (FSW) was modified to include a check on the altitude change - if the altitude is increasing, the radar must be locked onto the heatshield falling away from the lander instead of the ground closing in on the lander. However, the radar test program uncovered an even more insidious and difficult to resolve interaction. Under certain very specific conditions, the high-fidelity simulations showed the radar erroneously classifying a return signal from the heatshield as a return signal from the ground. In response to this mis-classification, the radar would return an altitude that was a full $5 \mathrm{~km}$ lower than the true altitude, and decreasing instead of increasing - thus fooling our heatshield lockup mitigation. An error of that magnitude would lead to certain mission failure.

The first response by the team was to delay the power on of the landing radar by an additional $40 \mathrm{sec}$ to increase separation between the lander and the heatshield. However, timeline changes alone could not remove the vulnerability - the radar firmware had to be modified, a mere three months prior to launch. The updated radar firmware pushed the region of vulnerability outside of the expected Phoenix trajectory.

\section{E. Turn to Entry and Cruise Stage Separation}

In 2006, trajectory analysis raised concerns about a potential for cruise stage re-contact with the Phoenix entry vehicle during flight through the atmosphere of Mars. At the time, as shown in Fig. 2, the baseline sequence of events included a turn to entry attitude seven minutes prior to entry, and separation of the cruise stage five minutes 
prior to entry. With the entry vehicle and cruise stage attitudes both aligned with the entry trajectory at the time of separation, the cruise stage simply follows the entry vehicle into Mars' atmosphere on the same trajectory. Only the separation rate produced by the separation event establishes a distance between the two vehicles during the five minutes between separation and atmospheric interface. Once in the atmosphere, the difference between the vehicle ballistic coefficients (Beta) creates divergence in their paths.

A thorough analysis of the potential for recontact of the vehicles included establishing a cruise stage break-up timeline through aerothermal methods, and understanding the trajectory of the resulting pieces from the break-up. This analysis established the potential for break-up pieces with high ballistic coefficients to overtake and impact the Phoenix entry vehicle. Analysis showed a catch-up and overtake miss distance as low as $6 \mathrm{~m}$. Given the uncertainty in the analysis, $6 \mathrm{~m}$ can be considered an impact.

An immediate solution to the issue might seem to be a repositioning of cruise stage separation earlier in the Phoenix timeline allowing the vehicles more time to achieve a larger and safe separation distance prior to entry into the atmosphere. However, because the Phoenix entry vehicle is powered by a battery of fixed capacity, loss of the solar array charging capability on the cruise stage after separation causes a negative battery power state by the time of landing. An early separation within the power limitation of the battery did not provide a reliable solution.

The simple design of the Phoenix landing system uses a single reaction control system (RCS) packaged on the lander and exited through the aeroshell. This RCS system is used both during the cruise to Mars and during EDL. Because the system is part of the lander system itself, the option to turn the entry vehicle to entry attitude after cruise stage separation existed, and indeed this is the solution chosen by the Phoenix engineering team. Prior to the turn to entry, the Phoenix spacecraft is in the sun point attitude keeping its solar arrays in full sunlight. This attitude is $46^{\circ}$ from the entry attitude. By separating the cruise stage first before performing the turn to entry, the cruise stage is given a component of velocity in the cross-trajectory direction by the separation event. The results of a break-up analysis for this scenario are shown in Fig. 5, which plots the separation distance time history between the cruise stage and the entry vehicle. The closest approach by an overtaking, high ballistic coefficient piece of the cruise stage is observed to be at a distance greater than $200 \mathrm{~m}$ from the entry vehicle. This result was deemed a sufficient outcome for the Phoenix mission, and thus the baseline was changed such that the cruise stage is first separated at seven minutes prior to entry, followed by a turn to entry attitude that completes by five minutes prior to entry.

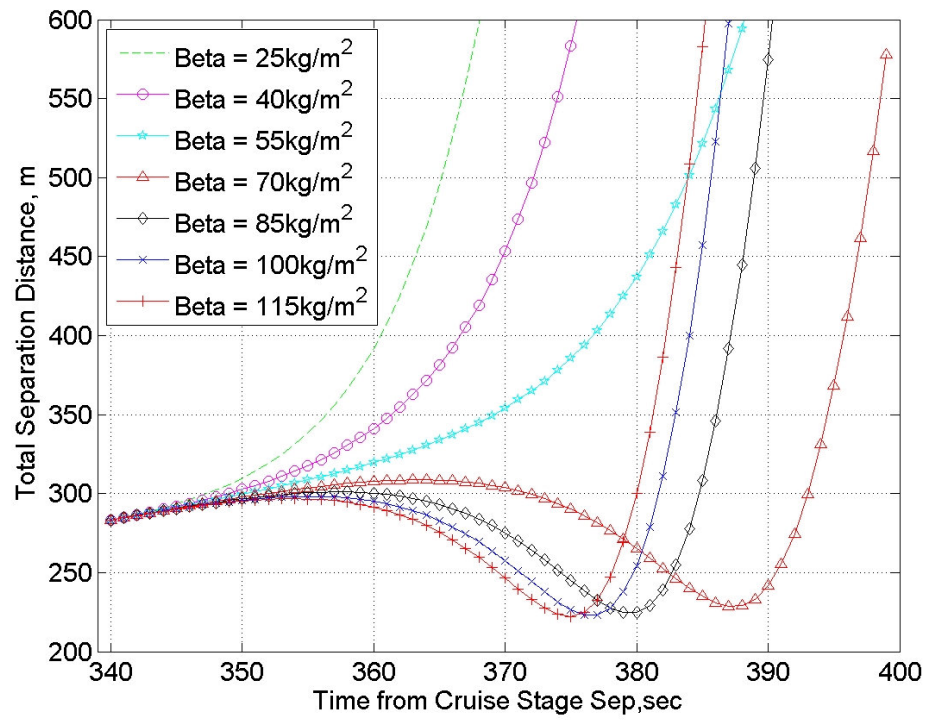

Figure 5. Cruise stage re-contact separation distances for separation in sun point attitude.

\section{F. Addition of the Backshell Avoidance Maneuver}

A failure of high regret on landing day would be to successfully execute a landing only to have the parachute follow the lander to the ground and drape the lander, effectively ending the mission. The backshell avoidance maneuver (BAM) was added to the Phoenix EDL architecture to avoid such a scenario.

American Institute of Aeronautics and Astronautics 


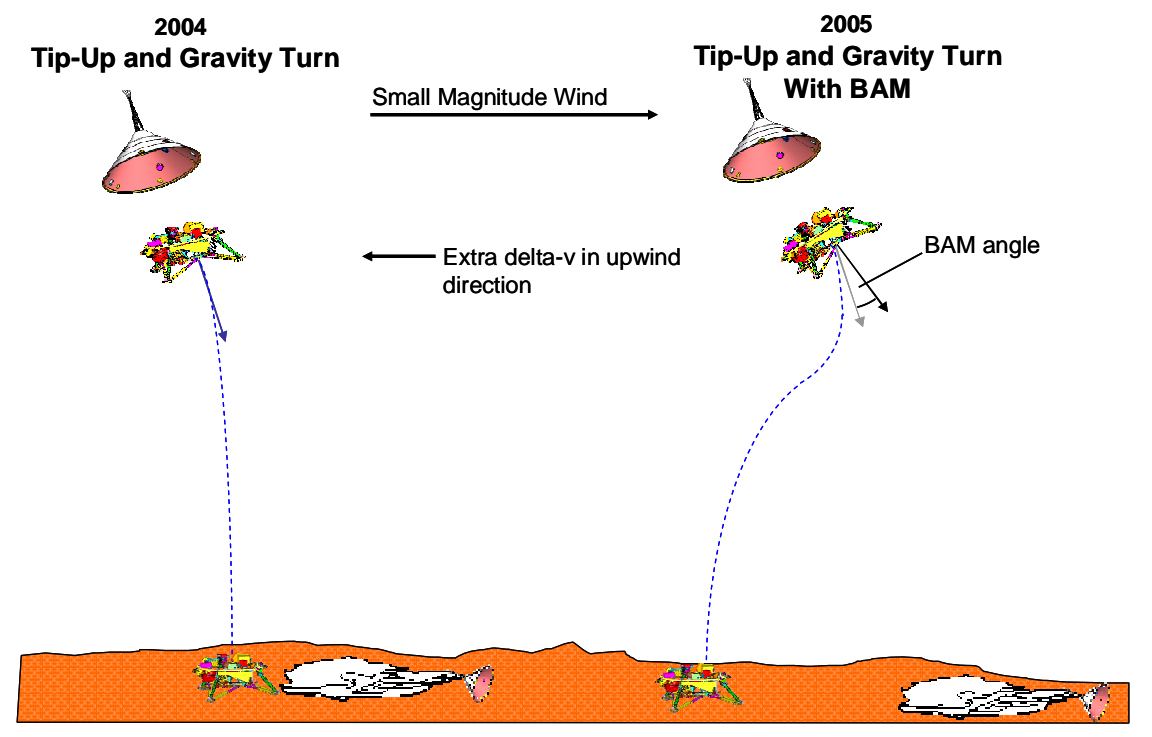

Figure 6. Backshell avoidance maneuver.

In cases of low wind or no wind at altitudes below $1 \mathrm{~km}$, the gravity turn powered descent performed by the Phoenix lander effectively becomes a straight down vertical descent. Having separated from the lander at about 1 $\mathrm{km}$, with effectively no wind, the parachute/backshell system will follow the lander to the ground landing on or very near it. This scenario is shown on the left side of Fig. 6. In higher wind cases at separation from the parachute/backshell, the combined system will have a horizontal velocity at or near the wind velocity. By definition, the gravity turn descent will arrest the lander's horizontal velocity bringing it to zero upon landing. The parachute/backshell, however, will retain the horizontal velocity and move downwind away from the lander. This situation provides an inherent mitigation of parachute draping risk. The BAM is designed to provide horizontal separation velocity when none or too little is provided by wind. This mitigation is achieved by augmenting the attitude tip-up maneuver that occurs three seconds after lander separation. Without the BAM, this tip-up is used to align the lander's thrust direction with the lander's velocity in preparation to execute the gravity turn. However, when the separation velocity is below a BAM threshold velocity, the tip-up maneuver is augmented to rotate beyond the velocity direction thereby adding horizontal velocity to the lander. This added velocity takes the lander upwind of the parachute and provides separation to prevent parachute draping of the lander. This scenario is shown on the right side of Fig 6.

\section{G. Thruster Efficacy and Change in Attitude Control Deadbands}

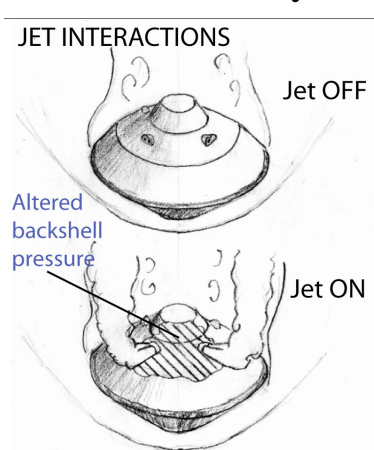

Jets can alter pressure on backshell, resulting in different control moments than intended

Figure 7. Thruster efficacy.

Approximately nine months before launch, an issue arose concerning the effectiveness of the RCS thrusters when used in flight through the atmosphere. These thrusters are used for pitch, yaw and roll attitude control from start of EDL to parachute deployment. The behavior of the thrusters themselves was not at issue, rather it was the impact of the thruster plumes on the aerodynamic flowfield surrounding the entry vehicle during hypersonic and supersonic flight that was of concern. Figure 7 illustrates the issue. As shown in the illustration, the RCS thruster exits are located on the backshell of the entry vehicle. When the thrusters are fired inside the atmosphere and at supersonic and hypersonic velocities, the thruster plume modifies the aerodynamic flowfield around the entry vehicle, which alters the pressure on the surface of the backshell. For Phoenix, Computational Fluid Dynamics (CFD) analysis showed that the modification of pressure resulted in a resultant torque on the vehicle that was opposite in direction from the expected torque generated from firing the RCS thruster. ${ }^{2}$ This situation is obviously undesirable. For the Phoenix configuration, CFD analysis was not able to resolve with certainty the magnitude of this effect. The minimum impact of the effect was a resulting smaller net torque generated by firing a thruster than desired. However, for the yaw axis in particular for Phoenix, CFD analysis could not rule out the possibility of a resulting net torque in the 
opposite direction from the desired torque. This situation could result in torque reversal, which if experienced for a prolonged period of time, could cause the attitude control system to drive the entry vehicle to undesirable attitude oscillations.

Faced with the limitations of the analysis techniques to clearly show the expected net torque on the entry vehicle resulting from the use of the RCS system while in the atmosphere, the Phoenix engineering team could not come to a crisp technical solution. However, trajectory analysis did show that, with tighter control at the top of the trajectory prior to the regime of concern, the Phoenix entry vehicle did possess sufficient inherent aerodynamic stability to traverse the flight through the atmosphere and meet required conditions at parachute deployment without use of the RCS system, and without passively spinning as did the MER and Pathfinder entry vehicles. The strategy for use of the RCS system then became that of a safety net. The control deadbands on the attitude controller were widened so that only in extreme situations, when the entry vehicle attitude behavior approaches limits of spacecraft capability, would the RCS system be used to dampen attitude behavior. Table 2 shows the attitude and attitude rate deadbands both for the initial Phoenix design and for the as-flown values developed after the thruster efficacy issue led to a change.

Table 2. Comparison of Initial Attitude Deadband Design vs. As-Flown Deadband Design.

\begin{tabular}{|c|c|c|c|c|c|c|c|c|c|c|c|c|}
\hline \multirow{3}{*}{ EDL Sub-Phase } & \multicolumn{6}{|c|}{ Attitude (deg) } & \multicolumn{6}{|c|}{ Attitude Rate (deg/s) } \\
\hline & \multicolumn{3}{|c|}{ Initial Design } & \multicolumn{3}{|c|}{ As Flown } & \multicolumn{3}{|c|}{ Initial Design } & \multicolumn{3}{|c|}{ As Flown } \\
\hline & $\begin{array}{l}\overline{\overline{0}} \\
\check{\simeq}\end{array}$ & $\frac{5}{\frac{5}{0}}$ & & $\begin{array}{l}\overline{\bar{o}} \\
\check{\Upsilon}\end{array}$ & $\frac{\frac{c}{0}}{\frac{0}{2}}$ & 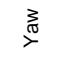 & & 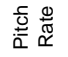 & & 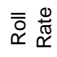 & 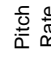 & 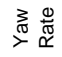 \\
\hline (Start at E-5min) & 1.7 & 5.0 & 5.0 & 1.0 & 2.0 & 1.0 & 0.03 & 1.0 & 1.0 & 0.05 & 0.2 & 0.05 \\
\hline Hypersonic 1 (Start at E-1min) & 1.7 & 5.0 & 5.0 & 1.0 & 2.0 & 1.0 & 0.03 & 1.0 & 1.0 & 0.05 & 0.2 & 0.05 \\
\hline Hypersonic 2 (Start at $0.294 \mathrm{~m} / \mathrm{s} 2$ ) & 1.7 & 2.0 & 2.0 & $\inf$ & 15.0 & 15.0 & 0.03 & 1.0 & 1.0 & 20.0 & 20. & 20.0 \\
\hline Hypersonic $3(5.3 \mathrm{~km} / \mathrm{s}$ & 1.7 & 10.0 & 10.0 & $\inf$ & 15.0 & 15.0 & 0.03 & 50.0 & 50.0 & 20.0 & 20. & 20.0 \\
\hline Hypersonic $4(3.8 \mathrm{~km} / \mathrm{s}-1.0 \mathrm{~km} / \mathrm{s})$ & 1.7 & 10.0 & 10.0 & inf & 15.0 & 15.0 & 0.03 & 50.0 & 50.0 & 20.0 & 20. & 20.0 \\
\hline Hypersonic $5(1.0 \mathrm{~km} / \mathrm{s}-0.62 \mathrm{~km} / \mathrm{s})$ & 1.7 & 5.0 & 5.0 & $\inf$ & 15.0 & 15.0 & 0.03 & 0.5 & 3.0 & 20.0 & 20. & 20.0 \\
\hline (Start at $0.62 \mathrm{~m} / \mathrm{s}$ ) & 1.7 & 5.0 & 5.0 & inf & 15.0 & 15.0 & 0.03 & 0.5 & 3.0 & 20.0 & 20. & 20.0 \\
\hline
\end{tabular}

\section{As Flown Architecture}

The preceding sections provided an overview of the significant changes made to the Phoenix EDL architecture. Figure 8 outlines the resulting as-flown architecture. Comparison of Fig. 8 with Fig. 2 illustrates the modifications that were required from the Mars 2001 Lander to the final flight day process. On May 25, 2008 this ballistic, balanced architecture provided a very successful powered landing on the northern plains of Mars. Reference 3 provides an overview of the reconstruction analysis performed for the actual Phoenix entry, descent, and landing.

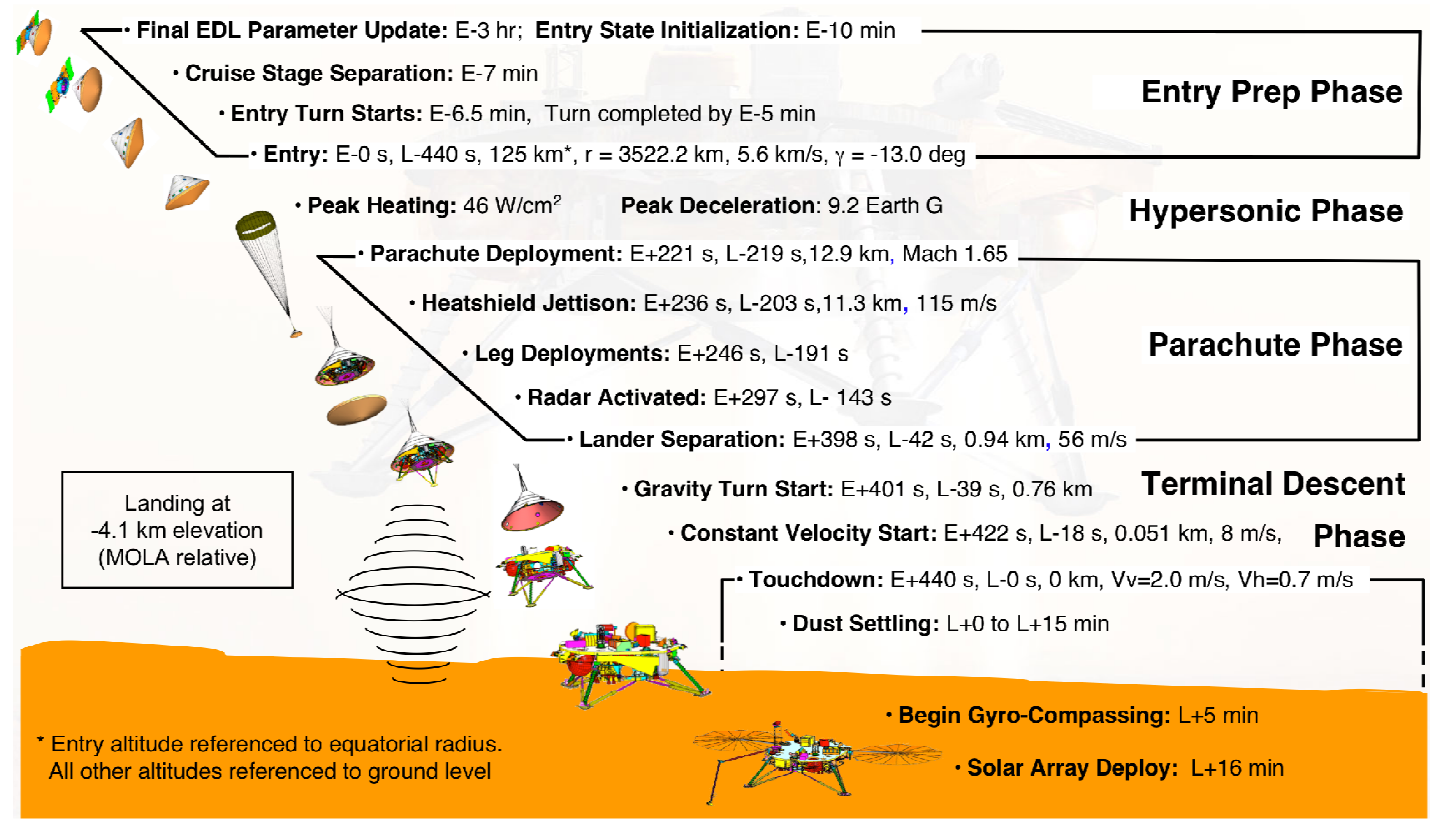

Figure 8. Final as-flown Phoenix EDL architecture.

American Institute of Aeronautics and Astronautics 092407 


\section{Conclusion}

Over the course of five years from the selection of Phoenix to landing day in 2008, the Phoenix EDL architecture went through a variety of changes. From simple changes driven by mission design differences to action taken in response to technical difficulties or robustness augmentation, major architectural changes were made. Mission design differences drove the entry velocity and landing site elevation, both of which proved less stressing to the EDL system than the original Mars 2001 Lander mission design. A desire for added robustness and simplification drove changes to both the parachute design and the parachute deployment algorithm, as well as the removal of hypersonic guidance and the addition of the backshell avoidance maneuver. The technical discovery of the RCS thruster efficacy issue led to a change in attitude control strategy. All these changes led to a robust EDL architecture that resulted in a successful landing on the northern plains of Mars.

\section{Acknowledgments}

The authors would like to acknowledge the contributions of the entire Phoenix EDL team at the Jet Propulsion Laboratory (Doug Adams, Erik Bailey, Gene Bonfiglio, Jim Chase, Curtis Chen, Dan Eldred, Richard Kornfeld, Paul Laufer, Mike Lisano, Rob Manning, Dana Runge, Dara Sabahi, David Skulsky, Cris Windoffer), at Lockheed Martin Space Systems (Tim Gasparrini, Brad Haack, Mark Johnson, Tim Linn, Tim Priser, Jay St. Pierre), and at NASA Langley Research Center (Jody Davis, Artem Dyakonov, Chris Glass, Karl Edquist, Ray Mineck, Jill Prince, Eric Queen, John Van Norman) for the overall success of the Phoenix landing. A portion of this research was carried out at the Jet Propulsion Laboratory, California Institute of Technology, under a contract with the National Aeronautics and Space Administration. Reference herein to any specific commercial product, process, or service by trade name, trademark, manufacturer, or otherwise, does not constitute or imply its endorsement by the United States Government or the Jet Propulsion Laboratory, California Institute of Technology.

\section{References}

${ }^{1}$ Shotwell, R. "Phoenix - The First Mars Scout Mission (A Midterm Report)," Acta Astronautica 57, July-October 2005 , pp. 121-134.

${ }^{2}$ Dyakonov, A. A., Glass, C. E., Desai, P. N., and Van Norman, J. W., "Aerodynamic Interference Effects Due to Reaction Control System for the Phoenix Entry Capsule," AIAA Paper 2008-7220, Proceedings of AIAA Guidance, Navigation, and Control Conference, Honolulu, HI, August 2008.

${ }^{3}$ Desai, P. N., Prince, J. L., Queen, E. M., and Grover, M. R., "Entry, Descent, and Landing Performance of the Mars Phoenix Lander" AIAA Paper 2008-7346, Proceedings of AIAA Guidance, Navigation, and Control Conference, Honolulu, HI, August 2008. 